\title{
RESEARCH PAPER \\ HISTOLOGICAL PATTERN OF LARYNGEAL CANCERS IN KOMFO ANOKYE TEACHING HOSPITAL
}

\author{
J. Opoku-Buabeng ${ }^{1}$ and O. Owusu-Afriyie ${ }^{2}$ \\ ${ }^{I}$ Department of Ear, Nose and Throat, School of Medical Sciences, KNUST, Kumasi \\ ${ }^{2}$ Department of Pathology, Komfo Anokye Teaching Hospital, Kumasi \\ Email: opokubuabeng75@yahoo.com
}

\begin{abstract}
Carcinoma of the larynx is one of the commonest malignant tumours of the upper respiratory system.The main objective of this study was to highlight the histological pattern and evaluate the occurrence of the malignant tumours of the larynx. This was a retrospective study involving 146 patients aged between 36 to 77 years with an average age of 59.7 years. Patients with symptoms and signs of laryngeal tumour were studied at the Komfo Anokye Teaching Hospital, Kumasi for a 10 year period from 2003 to 2012. Biopsies of suspected cases were taken and sent to the Histopathology Laboratory for histological analysis. The study revealed that there was a high occurrence of squamous cell carcinoma resulting in $92.0 \%$ of the patients. This was followed by verrucous carcinoma in $3.4 \%$ of the patients, adenocarcinoma and adenoid cystic carcinoma in $1.7 \%$ of the patients. Lastly, there was neuroendocrine carcinoma in only one patient (0.9\%). This study confirmed histologically that the squamous cell carcinoma was the most frequently manifested tumour of the larynx.
\end{abstract}

Keywords: Larynx, Cancers, Histology, Carcinomas

\section{INTRODUCTION}

Laryngeal cancer is the second most common type of head and neck cancer worldwide (Chu and Young, 2008). Laryngeal cancers comprised $2-5 \%$ of all malignant diseases diagnosed annually, with the male-to-female ratio of 5-20: 1 (Jemal et al., 2004). Epidemiological data suggest that the aetiology and pathogenesis laryngeal carcinomas are influenced by environmental and lifestyle-related factors such as tobacco use, alcohol consumption and exposure to toxic substances. In addition, dietary factors, irradiation, human papilloma virus infection and laryngopharyngeal reflux seemed to be significant carcinogenic factors.

The majority of laryngeal cancers are related to smoking and /or alcohol abuse. Heavy smoking and a low intake of vegetables and fruits increase the risk of laryngeal cancer 19- fold 
over that observed in non-smoking individuals who have a good intake of vegetables and fruits (Gallus et al., 2003).

Garavello et al. (2006) reported that the higher the percentage of alcohol consumption, the higher the incidence of developing laryngeal cancers. Edefonti et al. (2010) also reported in Milan (Italy) that the diets rich in animal products and animal fats are directly related and those rich in fruits and vegetables are inversely related to laryngeal cancer risk.

In the United States of America approximately 12,500 new cases are diagnosed each year (Parkin et al., 2005). Wünsch (2004) reported of around 8,000 new cases of laryngeal cancers occurring annually in the Brazilian population. In Canada 128 individuals in the province of British Colombia were identified as new cases in 2009 (BC Cancer Agency, 2012) whereas, 2,200 people in United Kingdom were diagnosed as laryngeal cancers in a year (Cancer back-up U.K., 2007). Cappellari (1997) reported of squamous cell carcinoma as being the most frequent histological type of laryngeal cancers.

Amusa et al. (2011) in Ile-Ife, Nigeria conducted a similar study by reviewing 13 cases of laryngeal cancers. The patients were aged 3888 years with a mean age of 69.9 years and the male -to- female ratio was 12:1. From their review, squamous cell carcinoma was identified in all the 13 cases. Iseh et al. (2011) also conducted a similar study in Sokoto (Nigeria) by reviewing 30 cases of laryngeal cancers. The male -to- female ratio was 5.7:1 and squamous cell carcinoma was identified in $66.7 \%$ of cases.

Olofsson and van Nostrand (1977), Del Negro et al. (2007), Zvrko and Golobović (2009) reported of sporadic cases of adenoid cystic carcinoma. This accounts for less than $1.0 \%$ of all malignant tumours of the larynx. Javadi et al. (2002) reported of a 12 year old boy with adenoid cystic carcinoma in Tehran (Iran). Ferlito and Recher (1980) reported in Padua (Italy) of an incidence rate of $3.2 \%$ of verrucous carcinoma. Varshney et al. (2004) also reported of an incidence rate of $(0.7 \%-1.0 \%)$ of verrucous carcinoma in all laryngeal cancers.

Even though not much has been reported in Ghana, a similar retrospective study was conducted on 115 patients at the Korle Bu Teaching Hospital (KBTH), Accra, from January, 1998 to December, 2003 (Kitcher et al. 2006). In their study, squamous cell carcinoma was identified in $88.7 \%$ of the cases, whereas adenocarcinoma was found in $1.7 \%$, spindle cell carcinoma in $1.7 \%$ and $6.1 \%$ were defined as unspecified.

This is the reason why this study was conducted to identify the histological pattern of laryngeal cancers within our environment.

\section{MATERIALS AND METHODS}

This was a retrospective study conducted at the ENT Department and the Pathology Department of the Komfo Anokye Teaching Hospital (KATH). The study involved the histological review of patients who had been diagnosed as cases of laryngeal cancer between January 2003 and December 2012 inclusive.

Data were obtained from the hospital records which included the age and gender, whilst their histological slides were reviewed. Also reviewed were histopathological reports received from other laboratories other than KATH. All the slides reviewed were stained with haematoxylin and eosin. The slides were read by the pathologist at the Pathology Department (KATH) using an Olympus Microscope BXO with a magnification power of (x 10-20). The data collected was entered into the Statistical Package for the Social Scientist (SPSS) 16.0 for Windows Product. The patients mean age, male -to-female ratio, gender percentages, the age group percentages and the histological presentation of the various slides were determined. 
Pattern of laryngeal cancers ... 18

\section{RESULTS}

In all 162 slides were retrieved but only 146 were employed for the study. The remaining sixteen slides were rejected because they were not technically well prepared. Of the rejected 16 slides four of them were from KATH whereas the remaining 12 were from other laboratories. Of the 146 review cases of laryngeal cancers, $122(83.6 \%)$ were males whereas $24(16.4 \%)$ were females and the male-tofemale ratio was 5.1: 1 as shown in Table 1.

Their ages ranged from 36 years to 77 years, with an average age of $59.7 \pm 3.2$ years (mean \pm standard deviation). With regard to their age group $2.7 \%$ were between 36 to 40 years, $4.8 \%$ were aged $41-45$ years, $8.9 \%$ were aged $46-50$ years, $16.4 \%$ were $51-55$ years old and $18.5 \%$ were $56-60$ years. With the rest of the patients $21.9 \%$ were $61-65$ years, $13.1 \%$ were $66-70$ years, $9.6 \%$ were $71-75$ years whereas $4.1 \%$ were 76-80 years as shown in Table 2 .

With respect to the 146 slides involved in the study $140(95.9 \%)$ were prepared in KATH Laboratory whereas the remaining $6(4.1 \%)$ were from other laboratories. The review of the slides and other histological reports from KATH and other laboratories revealed that squamous cell carcinoma resulted in 132 cases

Table 1: Gender distribution

\begin{tabular}{lcc}
\hline Gender & Frequency & Percentage (\%) \\
\hline Male & 122 & 83.6 \\
Female & 24 & 16.4 \\
Total & $\mathbf{1 4 6}$ & $\mathbf{1 0 0}$ \\
\hline
\end{tabular}

Male-to-female ratio 5.1:1

Table 2: Age distribution

\begin{tabular}{ccc}
\hline Age group (years) & Frequency & Percentage (\%) \\
\hline $36-40$ & 4 & 2.7 \\
$41-45$ & 7 & 4.8 \\
$46-50$ & 13 & 8.9 \\
$51-55$ & 24 & 16.4 \\
$56-60$ & 27 & 18.5 \\
$61-65$ & 32 & 21.9 \\
$66-70$ & 19 & 13.1 \\
$71-75$ & 14 & 9.6 \\
$76-80$ & 6 & 4.1 \\
\hline
\end{tabular}

Mean age $59.7 \pm 3.2$ years (Mean \pm Standard deviation) 


\section{Opoku Buabeng and Owusu-Afriyie}

$(90.3 \%)$, verrucous carcinoma in $6(4.1 \%)$, adenocarcinoma and adenoid cystic carcinoma in 3 $(2.1 \%)$ cases whereas neuroendocrine carcinoma resulted in $2(1.4 \%)$ cases as shown in Table 3.

All those prepared from other laboratories resulted as squamous cell carcinoma.

\section{DISCUSSSIONS}

Conventional squamous cell carcinoma is the most common histological type of laryngeal cancers. Nevertheless, it is important to be cognizant of the less common laryngeal malignancies because of their clinical behaviour and the concomitant implications for therapy.

Laryngeal cancer was most frequent in males than in females. In this study, there was a male -to-female ratio of 5.1:1, whereas Amusa et al. (2011) reported of 12:1, Iseh et al.(2011) also reported of 5.7:1 whereas Jemal et al. (2004) also reported of 5-20:1. The higher incidences in males than females may be due to the fact that males are more used to alcohol abuse and smoking than females which are some of the contributory factors in the development of laryngeal cancers in our environment. The average age of the patients in this study was 59.7 years, Amusa et al. (2011) also reported of 69.9 years in their study. This showed that laryngeal cancers often manifested in the fifth to sixth decade of life.

In this study squamous cell carcinoma was identified in $90.3 \%$ of all the cases, whereas Kitcher et al. (2006) reported of an incidence of $88.7 \%$, Amusa et al. (2011) identified $100 \%$ in their study and Iseh et al. (2011) also identified $66.7 \%$.

In this study verrucous carcinoma was the second commonest histological presentation of laryngeal cancers. This condition occurred in $4.1 \%$ of the cases, whereas Ferlito and Recher (1980) reported of an incidence rate of $3.2 \%$ and Varshney et al. (2004) also reported of an incidence rate of (0.7-1.0) \%.

Adenocarcinoma and adenoid cystic carcinoma were each identified in $2.1 \%$ of the cases whereas Kitcher et al. (2006) also reported of an incidence of $1.7 \%$. Olofsson and van Vostrand (1977), Del Negro et al. (2007), Zvrko and Golobović (2009) all reported of an incidence rate of less than $1.0 \%$ in their respective studies. This supports the fact that adenocarcinoma and adenoid cystic carcinoma are of rare occurrence.

\section{CONCLUSION}

Laryngeal cancer constitutes 2-5\% of cancers of the head and neck and is the second most common cancer. There are various histological

Table 3: Histological pattern of laryngeal cancers

\begin{tabular}{lcc}
\hline Histological pattern & Frequency & Percentage (\%) \\
\hline Squamous cell carcinoma & 132 & 90.3 \\
Verrucous carcinoma & 6 & 4.1 \\
Adenocarcinoma & 3 & 2.1 \\
Adenoid cystic carcinoma & 3 & 2.1 \\
Neuroendocrine carcinoma & 2 & 1.4 \\
\hline
\end{tabular}


types of laryngeal cancers but squamous cell carcinoma seemed to be the most predominant. Laryngeal cancers are more associated with certain social factors and addictions. Excessive use of alcoholic beverages, smoking and consumption of animal products and fats may be some of the risk factors whereas fruits and vegetables have been found to reduce the risk of developing laryngeal cancers.

\section{REFERENCES}

Amusa, Y. B., Badamus, A., Olabanji, J. K. and Ojebamiji, E. O. (2011). Laryngeal carcinoma: experience in Ile-Ife, Nigeria. Nigerian Journal of Clinical Practice. 14 (1): 748. Doi : 10. 4103/ 1119-3077. 79268.

Cappellari, J. O. (1997). Histology and pathologic prognostic indicators. Otolaryngologic Clinics of North America. 30(2): 251-68.

Chu, E. A. and Young, J. K. (2008). Laryngeal cancer: diagnosis and pre-operative work-up. Otolaryngologic Clinics of North America. 41: 673-695.

Del Negro, A., Ichihara, E., Tincani, A. J., Altemani, A. and Martins, A. S. (2007). Laryngeal adenoid cystic carcinoma: case report. Sao Paolo Medical Journal, 125(5): 295-6.

Edefonti, V., Bravi, F., Garavello, W., La Vecchia, C., Parpinal, M. and Francheschi, S. (2010). Cancer Epidemiology, Biomarkers \& Prevention. 19(1): 18-27.

Ferlito, A. and Recher, G. (1980). Ackerman's tumour (verrucous carcinoma) of the larynx: a clinicopathologic study of 77 cases. Cancer. 46(7): 1617-30.

Gallus, S., Bosetti, C. and Franceschi, S. (2003). Laryngeal cancer in women: tobacco, alcohol, nutritional and hormonal factors. Cancer Epidemiology, Biomarkers \& Prevention. 12: 514-517.

Garavello, W., Bosetti, C., Gallus, S., Moso, L.
D., Negri, E., Franceschi, S. and La Vecchia, C. (2006). Type of alcoholic beverage and the risk of laryngeal cancer. European Journal of Cancer Prevention. 15(1): 69-73.

Iseh, K. R., Abdullahi, M. and Aliyu, D. (2011). Laryngeal tumours: Clinical pattern in Sokoto, Northwestern Nigeria. Nigerian Journal of Medicine. 20(1): 75-82.

Javadi, M., Bafrouee, F. M., Mohseni, M. and Asqhari, A. (2002). Laryngeal adenoid cystic carcinoma in a child: a case report. Ear, Nose and Throat Journal. 81(1): 34-5.

Jemal, A., Tiweri, R. C., Murray, T., Ghafoor, A., Sameuls, A., Ward, E., Feuer, E. J. and Thun, M. J. (2004). American Cancer Society. Cancer Statistics, CA Cancer Journal for Clinicians. 54(1): 8-29.

Kitcher, E. D., Yarney, J. Y., Gyasi, R. K. and Chayno, C. (2006). Laryngeal cancer at the Korle Bu Teaching Hospital Accra, Ghana. Ghana Medical Journal. 40(2): 45-49.

Olofsson, J. and van Nostrand, A. W. (1977). Adenoid cystic carcinoma of the larynx: a report of four (4) cases and a review of the literature. Cancer. 40(3): 1307-13.

Parkin, D. M., Bray, F. and Ferlay, J., (2005). Global cancer statistics, 2002. CA Cancer Journal for Clinicians. 55(2): 74-108.

Varshney, S., Singh, J., Saxena, R. K., Kaushal, A. and Pathak, V. P. (2004). Verrucous carcinoma of larynx. Indian Journal of Otolaryngology and Head and Neck Surgery. 56(1)

Wünsch, F. V. (2004). The epidemiology of laryngeal cancer in Brasil. Sao Paolo Medical Journal. 122(5): 188-94.

Zvrko, E. and Golobović, M. (2009). Laryngeal adenoid cystic carcinoma. Acta Otorhinolaryngologica Italica. 29(5): 279-82. 